\title{
TIPOLOGÍA DE LOS SISTEMAS NUMERALES DE LAS LENGUAS CHIBCHAS DE COSTA RICA
}

\author{
Mario Portilla
}

\begin{abstract}
RESUMEN
Esta investigación propone clasificar las lenguas chibchas habladas en Costa Rica según tres parámetros: el tipo básico de numeración, el lugar del numeral en la frase como determinante y la existencia de una correlación entre el numeral y ciertas clases génericas nominales. Además, estudia detalladamente, desde una perspectiva diacrónica, cuál pudo haber sido el tipo básico de numeración del antepasado común de estas lenguas.
\end{abstract}

\begin{abstract}
This article proposes a classification of the Chibchan languages spoken in Costa Rica based on three parameters: the basic type of numeration, the placement of the number in the phrase as determiner, and the existence of a correlation between the number and certain gender classes of nouns. Furthermore, diachronically, it speculates on possible origins of the basic type of numeration in these languages.
\end{abstract}

\section{Introducción}

El propósito de la presente investigación es ofrecer una caracterización tipológica de los sistemas numerales de las lenguas chibchas que se hablan actualmente en Costa Rica. Se han tomado en cuenta para ello las siguientes lenguas: el guatuso, el térraba, el boruca, el cabécar, el bribri, el guaimí y el bocotál .

En una posible caracterización tipológica de los sistemas numerales de las lenguas chibchas, se podrían tomar en cuenta los siguientes parámetros:

\section{I. $\quad$ El tipo básico de los sistemas de numeración}

Este se determina por la cantidad expresada por el numeral mayor simple. Aunque teóricamente cualquier cantidad numeral podría constituir una base de numeración, los tipos más comunes en las lenguas son los siguientes: el tetracimal de base cuatro, el quinario de base cinco, el senario de base seis, el decimal de base diez y el vigesimal de base veinte. La tipología correspondiente a las lenguas chibchas estudiadas será tratada ampliamente en el próximo apartado. 


\section{El lugar que el numeral ocupe en la frase como determinante}

Por ejemplo, si el determinante aparece antepuesto o pospuesto al nombre determinado, o bien, si puede aparecer en ambas colocaciones.

Las lenguas estudiadas se distribuyen de la siguiente manera:

a. con numeral antepuesto: boruca.

b. con numeral pospuesto: térraba, bribri, bocotá y guaimí.

c. con numeral antepuesto o pospuesto: guatuso y cabécar.

Una consideración diacrónica es que, de acuerdo con lo anterior, se podría suponer que los numerales originalmente en la familia chibcha, o por lo menos en las lenguas de la llamada rama meridional ístmica de la familia a la que pertenecen todas las lenguas consideradas con excepción del guatuso, se colocaban pospuestos al nombre determinado. Los casos del boruca, el guatuso y el cabécar podrían ser explicados quizás por una influencia del español, cuyos numerales se anteponen al elemento determinado.

III. La existencia de una correlación entre los numerales y ciertas clases genéricas de los nombres

Con la excepción del guatuso y del boruca, todas las demás lenguas presentan formativos de clasificadores nominales relacionados con los numerales. El análisis de esta correlación escapa, sin embargo, a los propósitos de este trabajo. Por otra parte, es interesante señalar que, de acuerdo con Constenla (1988), la misma existencia de clasificadores nominales relacionados con los numerales es una innovación históricamente posterior de aquellas lenguas que la poseen.

\section{Tipología del sistema básico de numeración}

A continuación, se considerará el primer aspecto de la caracterización tipológica propuesta respecto de los numerales en las lenguas estudiadas: el tipo básico de numeración.

Estas lenguas se agrupan en tres tipos:

\subsection{Lenguas de base quinaria}

Estas son el guatuso y el cabécar de Ujarrás. Estas lenguas poseen numerales simples solamente para las cantidades de uno a cinco (ver ejemplos en el apéndice 1). Para cantidades mayores, utilizan numerales compuestos que toman como base el concepto del numeral 'cinco'. Por ejemplo, en guatuso, el número 'seis' se expresa como o:tin anaka:ţa, literalmente, 'cinco uno', o sea, 'cinco + uno'; y 'siete' se expresa como o:tin pa:unka, literalmente 'cinco dos', o sea, 'cinco + dos'.

Actualmente, en cabécar de Ujarrás, aunque existe, por supuesto, un morfema simple para expresar el numeral 'cinco', cantidades superiores a este número, como 'seis', 'siete', 
etc., se expresan por medio de una frase que incluye el término 'mano'. Entonces, 'seis' se expresa como sá? hulá ?étká kî ta ?ékJá, literalmente, 'nuestra mano uno más tener uno', es decir, 'una mano más uno'; 'siete' se expresa como sá? huJá ?étká kî ta bóI, literalmente, 'nuestra mano uno más tener dos', es decir, 'una mano más dos', que es otra manera de expresar 'cinco más dos'.

Para fines comparativos, es interesante describir cómo se forman los numerales 'diez', 'once', 'quince', 'dieciséis' y 'veinte' en ambas lenguas.

En guatuso, el número 'diez' se expresa como pake:nepake:ne kuiin, literalmente, 'ambas manos'. En cabécar de Ujarrás, 'diez' se expresa de manera semejante, como sá? hulá bótku, literalmente, 'nuestra mano dos'. En ambos casos, se expresan los conceptos numéricos de 2 x 5 .

El número 'once' se expresa de la misma manera en ambas lenguas: $(2 \times 5)+1$. En guatuso, pake:nepake:ne ku:in anaka:ța, literalmente, 'ambas manos uno' y, en cabécar de Ujarrás, literalmente, 'nuestra mano dos más tener uno'.

El número 'quince', sin embargo, no se expresa exactamente de la misma forma en ambas lenguas, a pesar de que en los dos casos se tiene como base el número 'cinco'. En guatuso, 'quince' se expresa como pake:nepakene kuin o:tin, literalmente, 'ambas manos cinco', es decir, $(2 \times 5)+5$. En cabécar de Ujarrás, se expresa como sá? hulá båḑ̧ắl, literalmente, 'nuestra mano tres', es decir, $(3 \times 5)$.

El número 'dieciséis' presenta también, por lo anterior, ciertas diferencias. En guatu-

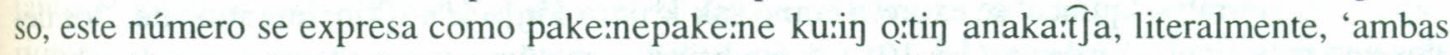
manos cinco uno' es decir, $(2 \times 5)+(5+1)$. En cabécar de Ujarrás, se expresa como sá? hulá bađđ̧ắ kí ta PékJá, literalmente, 'nuestra mano tres más tener uno', es decir, $(3$ x 5) + 1 .

Por último, el número 'veinte' se expresa en guatuso como pake:nepake:ne kuin nało:ki t̂a: mu:ri, literalmente, 'ambas manos y mis pies también', es decir, probablemente esta frase refleje la operación $(2 \times 5)+(2 \times 5)$. En cabécar, por su parte, 'veinte' se expresa como sá? hulá tkéd, literalmente, 'nuestra mano cuatro', es decir, 4 x 5 .

En este punto, es importante comentar el caso especial que representa el cabécar de Chirripó. En primer lugar, ilustra el principio teórico de que cualquier cantidad numérica puede convertirse en la base de un sistema de numeración. Dado que el informante del cual Bertoglia (1983) recogió los datos reconocía el número siete como la cantidad numérica mayor expresada por una forma simple, tomó este número como la base de numeración. En este caso, estaríamos frente a un sistema básico septenario. Por esta razón, por ejemplo, el número ocho se expresa como hkéli ki ékJá, literalmente 'siete más uno'.

Por otro lado, sin embargo, parece claro que este sistema de numeración constituye un desarrollo posterior de otros sistemas básicos de numeración. La misma investigadora que recopiló los datos manifiesta que su informante declaró que "las personas mayores empleaban un sistema distinto, basado en la expresión sá hulá (sic) pero subrayó el hecho de que este último sistema no es común entre la gente joven de Chirripó” (Bertoglia 1983: 10). Además, un análisis de los materiales de cabécar más antiguo permiten reconocer que las formas para los números 'seis' y 'siete', actualmente interpretados como formas numerales simples, provienen en realidad de formas compuestas. Así, por ejemplo, Lehmann en 1907 y 1908 (cf. Lehmann 1920) recoge los numerales 'seis' y 'siete' en cabécar de Chirripó de la siguiente forma: El número 'seis' aparece como 'skær-ki-ætka', o sea, ske् I kị ?étká, literalmente 'cinco más uno'. Obviamente, de esta forma compuesta provienen los vocablos actuales de cabécar de 
Chirripó hkiétku, tkétko, sétku. 'Siete' aparece como 'skær-ki-bótke', o sea, skéd kí bótku, literalmente, 'cinco más dos'. De esta frase provienen también las formas actuales de cabécar de Chirripó hké̃.l, tké̃.lka, sítku.

Por último, en los datos de Lehmann, la base numérica de cinco, en los números del seis al nueve, se expresa con la raíz del número 'cinco' ské I y no con la palabra para 'mano'.

\subsection{Lenguas de base decimal}

Estas lenguas son el térraba, el bribri y, quizá, el boruca. Estas presentan numerales simples del uno al diez (ver apéndice 1). Es interesante notar que, en térraba, el número 'diez' se podía expresar de dos formas: como $\mathrm{k}^{\mathrm{h}} \mathrm{uo}$-dbób, literalmente 'diez' igual que en bribri dabób, literalmente 'diez' y como sak khuará, literalmente 'los dedos (de las manos o de los pies, se supone) uno', es decir, 1 x 10 .

Los numerales mayores de 'diez' se expresan, en térraba y en bribri, por medio de formas compuestas y de forma semejante. Por ejemplo, en térraba, el número 'once' se expresa

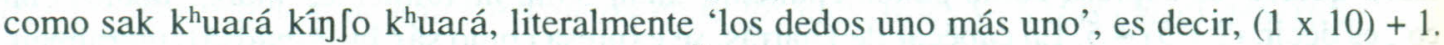
En bribri, 'once' se expresa como dabób êdz̧uk ki ěkuI, literalmente 'diez un-conjunto más uno', es decir, $(1 \times 10)+1$.

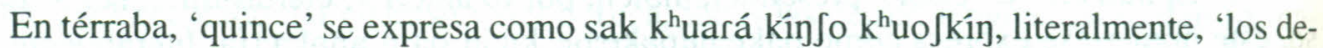
dos uno más cinco', es decir, $(1 \times 10)+5$. En bribri, este número se expresa como dabób êd3uk ki skêI, literalmente 'diez un-conjunto más cinco', es decir, $(1 \times 10)+5$.

En térraba, 'veinte' se expresa como sak фúk, literalmente 'los dedos dos', es decir, $2 \times 10$. En bribri, este número se expresa como dabób bûdz̧uk, literalmente 'diez dos-conjuntos', es decir, 2 x 10.

El boruca ha conservado solamente los numerales para expresar cantidades del uno al diez debido a su situación de extrema obsolescencia. En los datos recogidos en el siglo XIX, se encuentran apenas solamente los numerales del uno al ocho (cf. Quesada 1994). Por estas razones, es arriesgado incluso hasta clasificar el sistema que presenta esta lengua con unos datos tan exiguos como estos.

\subsection{Lenguas de base vigesimal}

Estas son el bocotá y el guaimí. Estas lenguas presentan raíces simples para los numerales del uno al diez. Por ejemplo, en bocotá, el número 'diez' para la clase de objetos largos se expresa como gada-táu, literalmente, 'diez'. En guaimí, 'diez' para la clase de objetos planos es kun-haudau, literalmente también, 'diez'.

En bocotá, 'once' se expresa como gada-táu gadá-de, literalmente 'diez uno', es decir, $10+1$. En guaimí, este número se expresa como kun-haudau biti kun-di, literalmente 'diez más uno'.

Además, ambas lenguas tienen una raíz simple para el número veinte. En bocotá, este número se expresa como gada-ri éde, literalmente 'veinte uno', es decir, 1 x 20 . En guaimí, 'veinte' se expresa como -gre, literalmente 'veinte'. 
La diferencia entre un sistema decimal y uno vigesimal se aprecia mejor por la base,utilizada en la formación de las decenas. Así, en térraba y en bribri, las decenas se forman multiplicando sobre la base de 'diez'. Por ejemplo, en térraba, 'cuarenta' se expresa como sak bkín, es decir, 4 x 10; 'cincuenta' como sak Jkín, es decir, 5 x 10. Por otra parte, en bocotá, 'cuarenta' se expresa como gada-hirí bu, es decir, 2 x 20 y 'cincuenta' como gada-hirí bu gada-tau, es decir $(2 \times 20)+10$.

Es interesante señalar, a modo de comparación, que el cabécar, de acuerdo con los datos recogidos por Lehmann en 1907, aparentemente era una lengua del tipo denominado quinario-vigesimal: 'cuarenta' se expresaba como 'sauak-bor', es decir, 2 x 20 y 'cincuenta' como sauak-mañár-ækala-moska', o sea, según Lehmann, 'tres veces veinte más la mitad de veinte", es decir, $(3 \times 20)+(20-10)$.

\section{Tipología del sistema numeral del protochibcha}

Con base en la descripción anterior, se pueden aventurar algunas consideraciones de índole diacrónica respecto del antecesor común a estas lenguas chibchas.

Se puede suponer que los datos expuestos apuntan a desechar la hipótesis de que estas lenguas fueran originalmente del tipo básico quinario. Una de las razones para ello es que tanto el guatuso como el cabécar utilizan distintas estrategias para expresar numerales superiores a cinco. Esta situación se puede ilustrar con el caso del numeral 'seis'. En guatuso, se expresa como 'el número cinco y el número uno; mientras que, en cabécar, se expresa como 'nuestra mano una vez más (tengo) el número uno'. Lo mismo sucede con el caso del número 'quince', que, como se ha dicho, es expresado en guatuso como $(2 \times 5)+5$ y en cabécar como $3 \times 5$.

Los textos antiguos tanto de cabécar como de guatuso dejan patente que en ambas lenguas existían diversas formas de expresar los numerales mayores de cinco. Para el guatuso, Lehmann reporta formas para 'seis' como 'tome mi mano y el número uno de mi otra mano'. Para el cabécar, ya se mencionó la estrategia para 'seis' de 'el número cinco más el número uno'.

Por otro lado, los datos tanto de Olabarrieta de finales del siglo XVIII (Fernández 1892), como los datos recogidos por Thiel (1882) y, sobre todo, los de Pittier (1904) muestran la existencia de formas simples para los numerales del seis al diez en algún dialecto de cabécar. Por ejemplo, Pittier recoge las formas 'ter-lu' para 'seis' y'dë-bop' para diez, las cuales tienen cognados en las demás lenguas chibchas comparadas.

Si se descarta, pues, la hipótesis de una base numeral original quinaria, queda la posibilidad de que el sistema numérico haya sido o bien de base decimal o bien de base vigesimal. Aunque a este respecto el autor no tiene todavía una certeza total, ciertos indicios hacen que prefiera la hipótesis de que el sistema era originalmente vigesimal.

Algunas consideraciones en las que se basa esta hipótesis son las siguientes:

Como se mencionó, el térraba utiliza también dos estrategias para expresar el numeral 'diez'. Una es el uso de la forma simple 'el número diez', otra es la forma compuesta 'los dedos (de las manos) una vez'. Como se vio, los numerales superiores a 'diez' usan sclamente como base la forma compuesta de 'diez'. El autor supone que esta forma compuesta es un vestigio del sistema vigesimal original. 
Además, es significativo que, entre ninguna de las lenguas que presentan numerales simples del uno al diez (térraba, bribri, boruca, bocotá y guaimí), se hallen cognados para los números 'ocho' y 'nueve'.

Por otro lado, si se parte de un sistema vigesimal como el del bocotá, por ejemplo, se podría explicar más fácilmente la aparición de las metáforas relacionadas con los dedos, las manos y los pies que ocurren en estas lenguas para expresar los numerales. Si veinte es la base original y esta base se relaciona con el cuerpo como totalidad, entonces puede ocurrir que 'veinte' sea 'los dos pies dos veces' como reporta Lehmann para el cabécar, o que 'diez' sea 'los dedos (de las manos o de los pies) una vez' como en térraba, o que 'cinco' sea 'una mano' como en cabécar. Precisamente, Lehmann reporta la forma 'saba ægela' (ækala) con el significado de 'nuestro cuerpo' para expresar también el número veinte en cabécar.

Igualmente, se podría hasta pensar que la anterior frase cabécar está relacionada con las raíces simples del numeral 'veinte' tanto del bocotá -hiri, como del guaimí -gre.

No hay que olvidar, además, que un sistema originalmente vigesimal pero no quinario, es decir, como el del bocotá o del guaimí, puede tomar naturalmente una subbase diez por ser la mitad de veinte para formar los numerales entre las decenas. Ello explicaría la similitud en la formación del número 'once' que existe, por ejemplo, entre el guaimí kun-haudau biti kun-di y el térraba sak k ${ }^{\mathrm{h}} u a$ aá kín $\int o \mathrm{k}^{\mathrm{h}}$ uará, expresados como $10+1$.

\section{Conclusiones}

$\mathrm{Al}$ pertenecer todas las lenguas indígenas de Costa Rica a la misma familia chibcha es posible considerar los diferentes tipos de sistema numeral desde una perspectiva diacrónica. Así, pues, el análisis de los datos presentado antes permite suponer, con bastante certeza, que el antepasado común a todas estas variedades no era de tipo quinario. Esto quiere decir que tanto el cabécar de Ujarrás como el guatuso representan una innovación tipológica al presentar un tipo numérico quinario. Como se ha dicho, el sistema septenario representado por el cabécar de Chirripó constituye ya un desarrollo posterior desde el sistema quinario original del cabécar.

Con menos certeza, se supone que el sistema numérico decimal del térraba, el bribri y el boruca constituye una modificación posterior de un sistema numérico vigesimal original. Sin embargo, existen dos razones fundamentales para sostener esta consideración: primero, que el térraba presenta dos estrategias diferentes para la formación del numeral 'diez' y que una de ellas, la compuesta, puede considerarse un vestigio de un sistema vigesimal original. Y, segundo, que los numerales 'ocho' y 'nueve' no encuentran cognados entre ninguna de las lenguas que presenta numerales simples del uno al diez (térraba, bribri, boruca, bocotá y guaimí).

Finalmente, se considera que el sistema vigesimal del bocotá y del guaimí representa mejor la posible situación original del protochibcha o, por lo menos, del antepasado común que dio origen a las variedades de la denominada rama meridional ístmica de la familia chibcha. 


\section{Notas}

1. Los datos para la clasificación han sido tomados de las siguientes fuentes: Bertoglia 1983 (cabécar), Constenla 1975 (guatuso), Fernández 1892 (cabécar, térraba), Gunn 1980 (guaimí), Lehmann 1920 (guatuso, térraba, cabécar, bribri), Margery 1982 (bribri), Pittier 1882 (térraba, cabécar), Quesada 1995 (boruca), Solís 1989 (bocotá), Thiel 1882 (térraba, cabécar).

\section{Bibliografía}

Bertoglia, Mafalda. 1983. "Los clasificadores nominales en los dialectos cabécares de Ujarrás y Chirripó”. Estudios de lingüística chibcha. 2: 3-13.

Constenla, Adolfo. 1975. La lengua guatusa: fonología, gramática y léxico. Tesis de licenciatura: Universidad de Costa Rica

1988. "Indicios para la reconstrucción de clasificadores en el sintagma nominal protochibcha." Revista de Filología y Lingüística de la Universidad de Costa Rica. 14(2): 111-8.

Fernández, León. 1892. Lenguas indígenas de Centro América en el siglo XVIII. San José: Tipografía Nacional.

Gunn, Robert. 1980. “Clasificación de los idiomas indígenas de Panamá con un vocabulario comparativo de los mismos". Lenguas Indígenas de Panamá. Tomo 7. Panamá: Instituto Nacional de Cultura.

Lehmann, Walter. 1920. Zentral-Amerika: Die Sprachen Zentral-Amerikas. Vol. 1. Berlín: Verlag Dietrich Reimer.

Margery, Enrique. 1982. Diccionario fraseológico bribri - español, español - bribri. San José: Editorial de la Universidad de Costa Rica.

Pittier, Henri. 1904. "Numeral systems of the Costa Rican Indians". American Anthropologist. 6(4): 451-2.

Quesada, Miguel. 1994."La lengua boruca en manuscritos del siglo XIX”. Estudios de lingüística chibcha. 13: 7-100.

1995. Hablemos boruca. San José: Ministerio de Educación Pública.

Solís, Mayra. 1989. "Los cuantificadores numerales en el bocotá de Chiriquî". Estudios de lingüística chibcha. 8: 141-52.

Thiel, Bernardo. 1882. Apuntes lexicográficos de las lenguas y los dialectos de los indios de Costa Rica. San José: Imprenta Nacional. 
Apéndice 1

Lista de numerales de las lenguas chibchas de Costa Rica

\begin{tabular}{|c|c|c|c|c|c|c|c|c|}
\hline & Térraba & Cabécar U. & Cabécar C. & Bribri & Bocotá & Boruca & Guatuso & Guaimí \\
\hline 1 & $k^{\text {huua-rá }}$ & Péklá,?étká & ?ékJá,?étká & ěkuI, ět & gadá-de,-éde & é?tse, é?tsi & anaka:t $\widehat{\text { a }}$ & kun-di \\
\hline 2 & -bó & ból & bó」 & bôl, bớtk & -bú, -u & bú?k & pa:unka & -bu \\
\hline 3 & -miá & bå̃dzád & bå̃dzád & 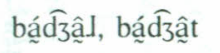 & -báai & man & po:iki:ri & - mau \\
\hline 4 & -bkín & tke्dé & $\begin{array}{l}\text { hkíl,tkílka, } \\
\text { tkị̂́tko }\end{array}$ & tkîl & -bága & báxkan & pa:keki:ri & -bogau \\
\hline 5 & $-\int k i ́ n$ & skeِd] & $\begin{array}{l}\text { hkéd,tkélka, } \\
\text { sịtku }\end{array}$ & skeِ & -tigá & fifkán & o:tin & -rike \\
\hline 6 & -tér & $\begin{array}{l}\text { sá? hulá Pétká } \\
\text { kî ta ?ék lá }\end{array}$ & $\begin{array}{l}\text { hkiétku, tkétko, } \\
\text { sétku }\end{array}$ & téru」 & -terége & tésan & o:tin anak:t Ja & $-\mathrm{ti}$ \\
\hline 7 & -kók & $\begin{array}{l}\text { sá? hulá ?étká } \\
\text { kí tạ bó.l }\end{array}$ & $\begin{array}{l}\text { hkéli, tkéli, } \\
\text { sotku }\end{array}$ & kûl & -gúge & kúx, kúxk & o:tin pa:unka & $-k i k i$ \\
\hline 8 & -kuón & $\begin{array}{l}\text { sá? hulá Pétká } \\
\text { kí tạ båḑåál }\end{array}$ & hkéli ki éklá & pákul & -hába & éxtan, (kúxtan) & o:tin po:iki:ri & -kuo \\
\hline 9 & - - kób & $\begin{array}{l}\text { sá? hulá ?étká } \\
\text { kí ta tké̃ I }\end{array}$ & hkéli kí ból & sư lítu, sũ.lítub & -áiga & kúxtan éxkux & o:tin pa:keki:ri & -inkon \\
\hline 10 & $\begin{array}{l}\text { khuo-dbób, } \\
\text { sak k khuará }\end{array}$ & sái hulá bótku & $\begin{array}{l}\text { hkéli kí } \\
\text { bådzád }\end{array}$ & dabób & gada-táu & $\begin{array}{l}\text { téxkux, } \\
\text { króftan, } \\
\text { bú?k kúx }\end{array}$ & $\begin{array}{l}\text { pake:ne pake:ne } \\
\text { ku:in }\end{array}$ & kun-haudau \\
\hline
\end{tabular}




\begin{tabular}{|c|c|c|c|c|c|c|c|c|}
\hline & Térraba & Cabécar U. & Cabécar C. & Bribri & Bocotá & Boruca & Guatuso & Guaimí \\
\hline 11 & $\begin{array}{l}\text { sak k kuará } \\
\text { kínjokhuará }\end{array}$ & $\begin{array}{l}\text { sá? hulá bótku } \\
\text { kí tå PékJá }\end{array}$ & hkéli kí hkíl & $\begin{array}{l}\text { dabób êđ̧̋ok } \\
\text { ki ěku」 }\end{array}$ & $\begin{array}{l}\text { gada-táu gadá- } \\
\text { de }\end{array}$ & & $\begin{array}{l}\text { pake:nepake:ne } \\
\text { ku:in anaka:t]a }\end{array}$ & $\begin{array}{l}\text { kun-haudau } \\
\text { biti kun-di }\end{array}$ \\
\hline 12 & $\begin{array}{l}\text { sak khuará } \\
\text { kínjo k kuobú }\end{array}$ & $\begin{array}{l}\text { sá? hulá bótku } \\
\text { kí tã bó }\end{array}$ & hkéJi kị hkêح́l & $\begin{array}{l}\text { dabób êđَ̧ok } \\
\text { ki bô! }\end{array}$ & $\begin{array}{l}\text { gada-táu godé- } \\
\text { bu }\end{array}$ & & $\begin{array}{l}\text { pake:nepake:ne } \\
\text { ku:in pa:unka }\end{array}$ & \\
\hline 15 & 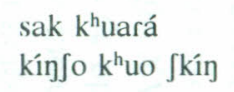 & $\begin{array}{l}\text { sá? hulá } \\
\text { båđđ̧ą́d }\end{array}$ & & $\begin{array}{l}\text { dabób êdžvk } \\
\text { ki skê」 }\end{array}$ & $\begin{array}{l}\text { gada-táu gada- } \\
\text { tigá }\end{array}$ & & $\begin{array}{l}\text { pake:nepake:ne } \\
\text { ku:in o:tin }\end{array}$ & \\
\hline 16 & $\begin{array}{l}\text { sak khuará } \\
\text { kíjJo k khuoter }\end{array}$ & $\begin{array}{l}\text { sá? hulá } \\
\text { bãdzáa } \\
\text { kí ta ?ékJá }\end{array}$ & & $\begin{array}{l}\text { dabób êđ̧uk } \\
\text { ki téruI }\end{array}$ & $\begin{array}{l}\text { gada-táu gada- } \\
\text { terége }\end{array}$ & & $\begin{array}{l}\text { pake:nepake:ne } \\
\text { ku:in o:tin } \\
\text { anaka:t]a }\end{array}$ & \\
\hline 20 & sak фớk & sá? hulá tkeِde & & dabób bûḑ̋ $v k$ & gada-ri éde & & $\begin{array}{l}\text { pake:nepake:ne } \\
\text { ku:in nało:ki } \\
\text { ţa: mu:ri }\end{array}$ & -gre \\
\hline 40 & sak bkin & & 'sauak-bor' & dabób tkîdzuvk & gada-hiri'bu & & & \\
\hline 50 & sak Jkín & & $\begin{array}{l}\text { 'sauak-mañár- } \\
\text { ækala-moska' }\end{array}$ & & $\begin{array}{l}\text { gada-hiri'bu } \\
\text { gada-táu }\end{array}$ & & & \\
\hline
\end{tabular}

